\title{
Clinical features and molecular modelling of novel MPZ mutations in demyelinating and axonal neuropathies
}

\author{
Paola Mandich ${ }^{*}$, , Paola Fossa ${ }^{2}$, Simona Capponi ${ }^{1}$, Alessandro Geroldi ${ }^{1}$, \\ Massimo Acquaviva ${ }^{1}$, Rossella Gulli ${ }^{1}$, Paola Ciotti ${ }^{1}$, Fiore Manganelli ${ }^{3}$, \\ Marina Grandis ${ }^{4}$ and Emilia Bellone ${ }^{1}$
}

\footnotetext{
${ }^{1}$ Department of Neuroscience, Ophthalmology and Genetics - Section of Medical Genetics, University of Genoa, Viale Benedetto XV, Genoa, Italy; ${ }^{2}$ Department of Pharmaceutical Sciences, University of Genoa, Viale Benedetto XV, Genoa, Italy; ${ }^{3}$ Department of Neurological Sciences, University 'Federico II' of Naples, Via Sergio Pansini 5, Naples, Italy; ${ }^{4}$ Department of Neuroscience, Ophthalmology and Genetics - Section of Neurology and Neurological Rehabilitation, University of Genoa, Genoa, Italy
}

Mutations in the myelin protein zero (MPZ) gene have been associated with different Charcot-MarieTooth disease (CMT) phenotypes, including classical demyelinating CMT1B and the axonal form of the disease (CMT2). The MPZ role in the pathogenesis of both demyelinating and axonal inherited neuropathies was evaluated in the Italian population by screening a cohort of 214 patients with CMT1 or CMT2. A MPZ mutation frequency of $7.9 \%$ in demyelinating cases and of $4.8 \%$ in axonal cases was observed. In the total cohort (264 patients), including those with mutations in other genes, a mutation frequency of $5.8 \%(7 / 121)$ in demyelinating cases and $4.2 \%(6 / 143)$ in axonal cases was found. Three novel MPZ mutations, two missense (p.Ser111Cys, p.Thr124Ala) and one frameshift (p.Tyr145fs) were found, and a molecular modelling approach was used to test the effects of these mutations on the protein structure. Electrostatic distribution changes within the protein, caused by the amino acid substitution, fit in with phenotypes presented by patients herein described. Our findings suggest that the clinical features associated with MPZ mutations depend partly on the nature of amino acid change and that molecular modelling may provide useful support, based on effects on secondary and tertiary protein structure, to predict the phenotype associated with MPZ mutations.

European Journal of Human Genetics (2009) 17, 1129-1134; doi:10.1038/ejhg.2009.37; published online 18 March 2009

Keywords: MPZ; CMT1; CMT2; molecular modelling

\section{Introduction}

Myelin protein zero (MPZ) is a $29 \mathrm{kDa}$ transmembrane glycoprotein, belonging to the immunoglobulin supergene

*Correspondence: Professor P Mandich, Department of Neuroscience, Ophthalmology and Genetics - Section of Medical Genetics, University of Genoa, c/o DIMI, Viale Benedetto XV, 6 - 16132 Genoa, Italy.

Tel: + 39010 3538960; Fax: + 39010 3538972;

E-mail: pmandich@unige.it

Received 1 October 2008; revised 27 January 2009; accepted 11 February 2009; published online 18 March 2009 family (IgCAM), uniquely expressed in Schwann cells. MPZ represents the major structural protein of the peripheral nervous system myelin. ${ }^{1}$

The final structure of the protein is characterised by three domains: ${ }^{2}$ a single immunoglobulin such as extracellular domain (ECD) of 124 amino acids, a single-span transmembrane domain (TD) of 26 amino acids and an intracellular C terminal domain (ICD) of 69 amino acids. Although ECD is highly hydrophobic, ICD has basic properties. Post-translational modifications occur as the 
protein is trafficked from the endoplasmic reticulum to the Schwann cell plasma membrane by the addition of a single N-linked oligosaccharide at Asn93, as well by the addition of the sulphate, acyl and phosphate groups. $^{3}$

Myelin protein zero acts as a homophilic intercellular adhesion molecule, involved in the formation and maintenance of the intraperiod and major dense lines of compact peripheral myelin. ${ }^{4}$ Coherently, when expressed in transfected non-glial cell lines, ${ }^{5,6} \mathrm{MPZ}$ promotes cellular aggregation, although transgenic mice, lacking MPZ expression, are affected by a severe demyelinating neuropathy with in compact myelin sheaths. ${ }^{7}$

Furthermore, a crystallographic study of recombinant ECD crystals $^{8}$ predicts that adjacent MPZ molecules interact in cis to form a lattice of homotetramers, which potentially adheres in trans to similar structures on opposing myelin loops. On the basis of this study, the interaction between MPZ homophilic tetramers is fundamental to the formation of the intraperiod line observed in compact myelin sheath. Recently, the combination of tetramer formation mediated by ECD and dimers mediated by the TD domain has been suggested as an alternative model for ECD packing in the myelin sheath. ${ }^{9}$

Myelin protein zero mutations, most affecting the P0 ECD, are associated with inherited peripheral neuropathies. More than 120 different mutations, both familial and sporadic, have been described and patients with MPZ mutations have been reported to show phenotypic heterogeneity, depending in part on the position and type of identified mutation (http://www.molgen.ua.ac.be/ CMTMutations). The clinical phenotypes associated with $M P Z$ mutations range from severe, congenital hypomyelination $(\mathrm{CH})$, Dejerine-Sottas syndrome (DSS) to late onset neuropathy with prominent axonal loss (Charcot-MarieTooth disease 2, CMT2). In addition, most MPZ mutations can be divided into two groups according to the age at onset and the clinical and pathological features. Mutations that disrupt the PO tertiary and quaternary structure are more likely to cause severe and early onset demyelinating forms. On the other hand, those mutations producing a subtly abnormal myelin sheath, which causes alterations in Schwann cell-axon interactions, are associated with late-onset mild axonal neuropathies. ${ }^{10}$ The molecular mechanisms underlying these phenotypes have been investigated in both mouse and cellular models. These models evaluated the effects of missense and deletion mutations, at late and early onset, on intracellular protein trafficking, glycosylation and intercellular adhesion. Mutations producing early onset neuropathy strongly affect protein trafficking and/or intercellular adhesion, whereas late onset mutations limit themselves to reduce the P0-mediated intercellular adhesion. ${ }^{11,12}$

Our study aimed at evaluating the $M P Z$ role in the pathogenesis of both demyelinating and axonal inherited neuropathies in the Italian population by screening a cohort of CMT1 and CMT2 patients. We describe three novel MPZ mutations, two missense (p.Ser111Cys, p.Thr124Ala) and one frameshift (p.Tyr145fs). To evaluate the effect of ECD mutations on the protein structure and to find a correlation with the clinical phenotype, we used a molecular modelling approach. Our findings confirm earlier studies based on different methods and provide a new technological tool, based on the effects of the secondary and tertiary protein structure, to predict the clinical features associated with $M P Z$ mutations.

\section{Materials and methods Patients}

Two hundred and fourteen consecutive blood samples from unrelated patients with peripheral motor and sensory neuropathy were enrolled at our Service of Medical Genetics for molecular diagnosis of CMT (both familial and isolated; $83 \mathrm{CMT} 1 / \mathrm{DSS}, 125 \mathrm{CMT} 2,6 \mathrm{CH}$ ). All patients signed a written informed consent. The phenotype was retrospectively defined on the basis of the clinical and electrophysiological data, and when available on sural nerve biopsy evaluation was reported by highly specialised Italian neurological centers. A CMT1 was diagnosed when the NCVs, recorded from the median/ulnar nerves, were $<38 \mathrm{~m} / \mathrm{s}$, whereas CMT2 was diagnosed when upper-limb NCVs were $>38 \mathrm{~m} / \mathrm{s}$ with reduced compound muscle action potential (cMAP). $\mathrm{CH}$ was diagnosed on sural nerve biopsy according to the described criteria. ${ }^{13}$

CMT1 patients had earlier tested negative for the CMT1A duplication and HNPP deletion, as well as for mutations in the coding regions of PMP22, GJB1 and EGR2. Mutations in the most common genes involved in axonal neuropathies (mitofusin-2, neurofilament light gene and small heat-shock proteins) were excluded in CMT2 patients.

\footnotetext{
Molecular analysis

Genomic DNA was extracted from peripheral blood samples according to a standard protocol. The coding sequence of $M P Z$ and the flanking exon-intron boundaries were amplified by polymerase chain reaction (PCR) using primer sequences published by Nelis et al. ${ }^{14}$ PCR products were analysed for mutations by denaturing high performance liquid chromatography (DHPLC Wave MD, Transgenomic). The melting profile for each DNA fragment, the respective elution profiles and column temperature were determined using the Wave Maker software from Transgenomic, with optimisation performed against a positive control. Each profile alteration was further investigated by direct sequencing on automated sequencer ABI Prism 3100Avant Genetic Analyzer (Applied Biosystems, Foster City, CA, USA).
} 


\section{Molecular modelling}

The three-dimensional structure coordinate file of the ECD of the P0 was obtained from the Protein Data Bank, PDB entry $1 \mathrm{NEU} .^{8}$ Mutations on the primary sequence were inserted by means of the Biopolymer module implemented in Sybyl (Sybyl 7.0 Tripos Inc. 1699 South Hanley, Missouri, 63144, USA). Partial atomic charges on the amino acid residues of interest were calculated by the GasteigerHuckel method. The software Sybyl was also used for the graphical visualisation of the protein.

\section{Results}

\section{Mutation analysis}

Myelin protein zero molecular analysis identified 13 heterozygous mutations (9 missense, 3 frameshift and 1 nonsense) out of 15 index cases (11 familial, 2 sporadic and 2 isolated). Two mutations affect the ICD, and all the remaining are within the ECD (Table 1).

One mutation was associated with $\mathrm{CH}$, one with DSS, five with CMT1, six with CMT2 (p.Thr124Met segregating in three families), thus providing a mutation frequency of $7.9 \%(7 / 89)$ in demyelinating cases and $4.8 \%(6 / 125)$ in axonal cases when only cases negative for mutations in the most common genes were considered. In the total cohort (264 patients), including those with mutations in other genes, a mutation frequency of $5.8 \%$ (7/121) in demyelinating cases and $4.2 \%(6 / 143)$ in axonal cases was found.

Three of these mutations were not described earlier: two missense mutations (p.Ser111Cys, p.Thr124Ala) and one frameshift (p.Tyr145fs) within the ECD. These mutations were not found in 200 normal controls. Patients with p.Ser111Cys and p.Thr124Ala mutations had a positive family history for CMT and the pathogenetic role of the variants identified have been confirmed by co-segregation in affected relatives; both variants affect two residues that are highly conserved in different species and, moreover, Thr124 is a hot spot for mutations in MPZ. ${ }^{15}$

Earlier described mutations and associated features in the carrying patients are overlapping those reported in the literature. The clinical and neurophysiological features of patients with novel mutations are summarised below.

Patient 1 (p.Ser111Cys) Patient with severe demyelinating neuropathy at early onset (first decade). Family history was positive for peripheral neuropathy (father affected and carrying the p.Ser111Cys variant). Neurological examination (at 32 years) showed severe distal muscle weakness and wasting in the lower limbs, as well as the bilateral pes cavus. NCVs are decreased both at the upper and lower limbs $(14.6 \mathrm{~m} / \mathrm{s}$ median nerve; $12 \mathrm{~m} / \mathrm{s}$ ulnar nerve; $7.5 \mathrm{~m} / \mathrm{s}$ posterior tibial nerve and no response was recorded from the sural nerve).

Patient 2 (p.Thr124Ala) Patient with axonal neuropathy at late onset (38 years). Family history was positive (mother and sister affected). Neurological examination (at 40 years) showed bilateral pes cavus and slight muscle weakness and wasting in the anterior compartment of the leg. No pupillary abnormalities were observed. NCVs are slightly decreased in the lower limbs $(39 \mathrm{~m} / \mathrm{s}$ posterior tibial nerve; $42 \mathrm{~m} / \mathrm{s}$ sural nerve) and normal in the upper limbs $(56.4 \mathrm{~m} / \mathrm{s}$ ulnar nerve; 48.5 radial nerve). cMAP amplitudes were all reduced.

Patient 3 (p.Tyr145fs) Patient with axonal neuropathy at late onset (at 40 years). Family history was negative. Neurological examination (41 years) showed bilateral pes cavus and muscle weakness and wasting prominent in the anterior compartment of the leg. Postural tremor of arms and hands was also present. NCVs are slightly decreased both in the upper and lower limbs $(38 \mathrm{~m} / \mathrm{s}$ median nerve; $32.5 \mathrm{~m} / \mathrm{s}$ posterior tibial nerve and $30 \mathrm{~m} / \mathrm{s}$ sural nerve). cMAP amplitudes were reduced.

Table 1 MPZ mutations in patients with peripheral sensory-motor neuropathy

\begin{tabular}{|c|c|c|c|c|c|c|}
\hline cDNA change & Protein change & Domain & Patient phenotype & Age at onset & Inheritance & Reference \\
\hline c. $152 C>T$ & p.Ser51Phe & ECD & СMT2 & 48 & $A D$ & CMTMutations/db \\
\hline c. $181 \mathrm{G}>\mathrm{A}$ & p.Asp61Asn & $\mathrm{ECD}$ & CMT1 & 1 & Isolated & Bellone et $a l^{16}$ \\
\hline c. $233 \mathrm{C}>\mathrm{T}$ & p.Ser78Leu & ECD & CMT1 & $>40$ & $\mathrm{AD}$ & Nelis et $a l^{14}$ \\
\hline c. $292 C>T$ & p.Arg98Cys & ECD & DSS & 1 & Sporadic & CMTMutations/db \\
\hline c. $293 G>A$ & p.Arq98His & ECD & CMT1 & $<10$ & $A D$ & CMTMutations/db \\
\hline c. $306 \mathrm{del} A$ & p.Val102fs & $\mathrm{ECD}$ & CMT2 & 40 & $A D$ & CMTMutations/db \\
\hline c. $327 \mathrm{~T}>\mathrm{A}$ & p.Asp109Glu & $\mathrm{ECD}$ & CMT2 & 60 & $A D$ & Santoro et $a l^{17}$ \\
\hline c. $332 C>G$ & p.Ser111Cys & $\mathrm{ECD}$ & CMT1 & $<10$ & $A D$ & This report \\
\hline c. $370 A>G$ & p.Thr124Ala & $\mathrm{ECD}$ & CMT2 & 39 & $A D$ & This report \\
\hline c. $371 \mathrm{C}>\mathrm{T}$ & p.Thr124Met & $\mathrm{ECD}$ & CMT2 & $38 ; 46 ; 54$ & $A D$ & CMTMutations/db \\
\hline c.432_433insT & p.Tyr $145 \mathrm{fs}$ & $\mathrm{ECD}$ & CMT2 & 40 & Isolated & This report \\
\hline c. $643 \bar{C}>T$ & p.G $\ln 215 X$ & $I C D$ & $\mathrm{CH}$ & 1 & Sporadic & CMTMutations/db \\
\hline c.699_702delTGAG & p.Ser233fs & ICD & CMT1 & $<10$ & $\mathrm{AD}$ & CMTMutations/db \\
\hline
\end{tabular}

Abbreviations: AD, autosomal dominant; ECD, extracellular domain; ICD, intracellular domain.

Isolated: no family history, parents not examined; Sporadic: no family history, parents examined and negative for the specific mutation; CMTMutations/db: http://www.molgen.ua.ac.be/CMTMutations. 


\section{Molecular modelling analysis}

Molecular modelling techniques were applied to evaluate at the molecular level the effects determined by the two novel ECD missense mutations on the protein structure. In addition, simulations of the electrostatic variations determined by amino acid changes were also performed for other two ECD mutations (p.Asp61Asn, p.Asp109Glu) reported earlier by our group (Santoro L et $a l^{17}$ personal communication).

p.Asp61Asn In the wild-type protein, this residue, negatively charged at neutral $\mathrm{pH}$, has acidic features and acts as a hydrogen bond acceptor (blue area, Figure 1a, left side), owing to its side chain carboxylic function. Differently, Asn61 is basic and may act both as a hydrogen bond acceptor (carboxylic group of the amidic function) and hydrogen bond donor (red area, Figure 1a, right side) based on its side-chain side amidic nitrogen. Thus, this mutation produces a polarity variation and consequently affects the hydrogen bonds net responsible for the correct folding and dimerisation of the protein. According to our in silico simulations, the replacement of Asp with Asn, its amide, might produce misfoldings in the $\mathrm{PO}$ protein caused by the need to counterbalance both the different polarity of the amidic portion and the bulkier side chain (total volume $91 \AA^{3}$ and $96 \AA^{3}$, respectively, for Asp and Asn).

p.Asp109Glu This amino acid change does not determine the dramatic effects in the electrostatic distribution of the protein region involved (Figure 1b) as Asp and Glu both display acidic features, with a carboxylic moiety on the side chain. In any case, the pattern of hydrogen bonding interactions in this area is slightly modified by the need for accommodating the longer side chain of Glu (total volume $109 \AA^{3}$ ), with some minor variations in the protein stability and folding.
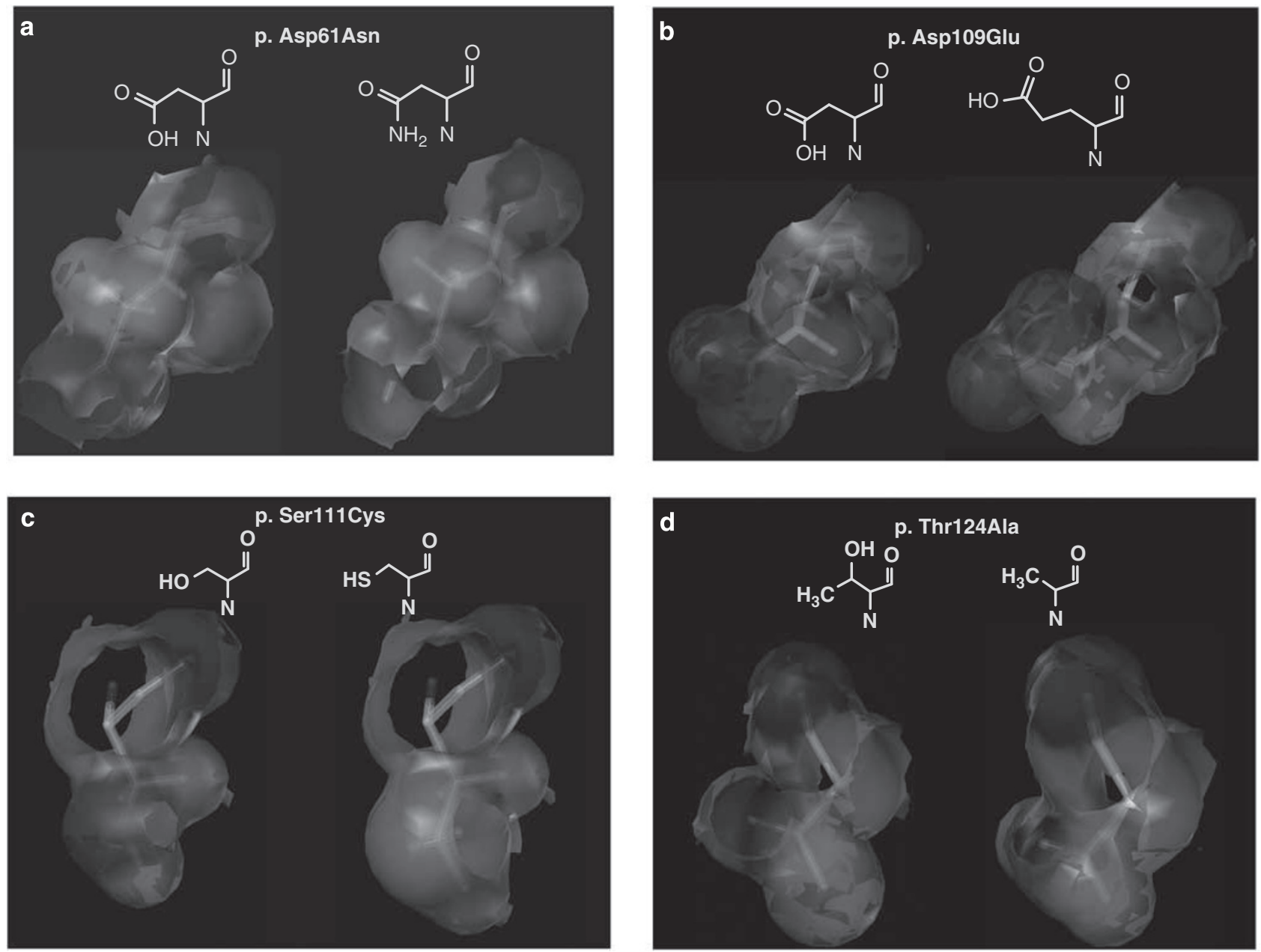

Figure 1 The different patterns of hydrogen-bonding features are highlighted. Blue area: hydrogen bond acceptor. Red area: hydrogen bond donor.(a) Substitution of residue p.Asp61Asn. (b) Substitution of residue p.Asp109Glu. (c) Substitution of residue p.Ser111Cys. (d) Substitution of residue p.Thr124Ala. The color reproduction of this figure is available on the html full text version of the manuscript. 
p.Ser111Cys This substitution, involving a polar residue, serine, whose hydroxyl group on the side chain is able to act as a hydrogen bond donor, determines a strong effect on the protein. The introduction in position 111 of a cysteine, a lipophilic residue, markedly lowers the polarity of the protein in this area (Figure 1c). As in the case of the Asp61Asn substitution, heavy variations in the hydrogen bonds net, responsible for the correct folding and dimerisation of the protein, and additional misfoldings necessary to pack the longer side chain of cysteine (total volume $86 \AA^{3}$ and $73 \AA^{3}$, respectively, for Cys and Ser) are expected. Moreover, according to our calculations, Cys111 is unlikely to form sulphur bridges with neighbour residues.

p.Thr124Ala In this mutation, a hydrophobic residue replaces a polar one but the change is likely to moderately effect the polarity of the protein compared with p.Ser111Cys change. Even if the X-ray of MPZ does not include this residue, molecular modelling studies support the hypothesis that the hydrogen bonds net will be modified by the substitution of the hydroxyl group of Thr124 (total volume $93 \AA^{3}$ ), a hydrogen bond donor group, with the smaller methyl group of Ala. However, the smaller volume of the new residue (total volume $67 \AA^{3}$ ) and its small degree of hydrophobicity, in comparison with, for example, a Leu or an Ile residue, probably affects to a lower extent the correct folding of the P0 protein. In addition, in Figure $1 \mathrm{~d}$ it is evidently a global similarity in the hydrogen bonding features of the two residues.

\section{Discussion}

Myelin protein zero mutations have been reported in association with both the demyelinating and axonal forms of CMT disease. Patients with MPZ mutations show clinically severe, moderate or mild neuropathies, depending partly on the specific mutation. Shy et al ${ }^{10}$ described the correlation between specific MPZ mutations and the neurophysiological and clinical phenotypes. Thus, patients can be divided into two groups: those who show an early onset and those with a late onset. The first ones are affected by a severe demyelinating CMT, most likely caused by mutations that distort the PO tertiary structure and interfere with the correct myelination during the development. On the other hand, the second group is affected by a mild-axonal CMT, probably originated by mutations that do not permit Schwann cell-axon interactions. The mutations p.Ser78Leu (named p.Ser49Leu in ${ }^{10}$ ) and p.Arg98His (named p.Ser69Leu in ${ }^{10}$ ) proved exceptions to the rule as they have been associated with both phenotypes. ${ }^{10}$ However, p.Ser78Leu was also found within our cohort, and it is associated with a very late onset CMT1 phenotype, thus further enlarging the phenotypic spectrum associated with this mutation.
Morphological studies of the early- and late-onset forms of CMT1B have shown distinct molecular and pathological abnormalities, ${ }^{18,19}$ and the molecular mechanisms underlying these differences have been investigated in mouse and cellular models. Wrabetz et al ${ }^{11}$ produced a transgenic mouse carrying two distinct $M P Z$ mutations, p.Ser63Cys and p.Ser63del, both causing a demyelinating neuropathy. The p.Ser63Cys mutation creates a packing defect in the myelin sheath, whereas p.Ser63del does not reach the myelin sheath and is retained in the endoplasmic reticulum where it elicits an unfolded protein response in Schwann cells. These data support the idea that the variability of MPZ-related phenotypes derives from different kinds of gain of abnormal function. Subsequently, Grandis et $a l^{12}$ investigated the molecular mechanisms underlying different $M P Z$ mutations by transient transfection in vitro models. They found that some late-onset mutations cause a partial loss of function. Alternatively, mutant proteins causing an early-onset neuropathy may either produce a mistrafficking of the P0, or reach the cell surface and cause a neuropathy by 'dominant negative' effects that alter the structure of myelin. In our series, clinical features in patients carrying already described mutations are overlapping with those reported in the literature. The p.Thr124Met was found in three unrelated families and associated with late-onset axonal neuropathy with pupillary signs. In one family, three patients carrying p.Thr124Met have been examined and two of them developed a Guillain-Barré syndrome.

Three mutations were not described earlier in the literature, p.Ser111Cys, p.Thr124Ala and p.Tyr145fs. The last mutation produces a premature stop codon at amino acid 148 and is associated with a late-onset CMT2. The frameshift could result in the synthesis of a non-functional protein that is truncated very early in the ECD and therefore cannot be properly inserted into the myelin membrane. Steck et $a l^{20}$ showed normal P0 expression levels and a normal protein size in the nerve of a heterozygous individual carrying a different frameshift mutation (p.Val102fs). The absence of the expected 50\% reduction in P0 levels suggests a compensatory mechanism driven by feedback signalling, in which increased expression of the normal allele can overcome the deficit, leading to near-to-normal levels of P0 in the myelin sheath. P0 expression patterns are in accordance with the mild phenotype shown by their patient.

Through a molecular modelling approach, this study evaluated the effect of novel missense mutations on the PO protein structure, providing a complementary approach to the study of mechanisms underlying MPZ-associated neuropathies. Molecular analysis has also been extended to two other ECD mutations found earlier in our cohort. As far as p.Asp109Glu and p.Thr124Ala are concerned, molecular modelling has not shown any important change on the electrostatic distribution in the protein region. On 
the contrary, both p.Asp61Asn and p.Ser111Cys severely affect the polarity of the affected region of the protein, leading to heavy variations in the hydrogen bond net, responsible for the correct folding and dimerisation of the protein. Additional misfolding in the PO protein is expected owing to a different steric hindrance produced by both amino acid changes.

Phenotypes presented by patients herein described correlate with changes in the electrostatic distribution in the protein, caused by the amino acid substitutions. Substitutions of p.Asp109Arg and p.Thr124Ala are associated with a mild, late-onset axonal neuropathy, whereas p.Asp61Asn and p.Ser111Cys substitutions are associated with severe early-onset demyelinating neuropathy. These findings confirm that the clinical features associated with MPZ mutations depend in part on the nature of the amino acid change. Molecular modelling, investigating the folding and the stability of the mutated proteins, offers useful technological support to unravel the effects of $M P Z$ mutation on the protein structure.

\section{Acknowledgements}

We are grateful to the patients, their families and the ACMT-Rete association for participation in the studies and for ongoing support.

\section{References}

1 Spiryda LB: Myelin protein zero and membrane adhesion. J Neurosci Res 1998; 54: 137-146.

2 Lemke G, Axel R: Isolation and sequence of a cDNA encoding the major structural protein of peripheral myelin. Cell 1985; 40: 501-508.

3 Eichberg J: Myelin P0: new knowledge and new roles. Neurochem Res 2002; 27: 1331-1340.

4 Martini R, Mohajeri MH, Kasper S et al: Mice doubly deficient in the genes for $\mathrm{PO}$ and myelin basic protein show that both proteins contribute to the formation of the major dense line in peripheral nerve myelin. J Neurosci 1995; 15: 4488-4495.

5 Filbin MT, Walsh FS, Trapp BD, Pizzey JA, Tennekoon GI: Role of myelin P0 protein as a homophilic adhesion molecule. Nature 1990; 344: 871-872.

$6 \mathrm{Xu} \mathrm{W}$, Shy M, Kamholz J et al: Mutations in the cytoplasmic domain of $\mathrm{PO}$ reveal a role for PKC-mediated phosphory- lation in adhesion and myelination. I Cell Biol 2001; 155: 439-446.

7 Giese KP, Martini R, Lemke G, Soriano P, Schachner M: Mouse P0 gene disruption leads to hypomyelination, abnormal expression of recognition molecules, and degeneration of myelin and axons. Cell 1992; 71: 565-576.

8 Shapiro L, Doyle JP, Hensley P, Colman DR, Hendrickson WA: Crystal structure of the extracellular domain from P0, the major structural protein of peripheral nerve myelin. Neuron 1996; 17: $435-449$.

9 Plotkowski ML, Kim S, Phillips ML, Partridge AW, Deber CM, Bowie JU: Trasmembrane domain of myelin protein zero can form dimers: possible implications for myelin construction. Biochemistry 2007; 46: 12164-12173.

10 Shy ME: Peripheral neuropathies caused by mutations in the myelin protein zero. J Neurol Sci 2006; 242: 55-66.

11 Wrabetz L, D'Antonio M, Pennuto $M$ et al: Different intracellular pathomechanisms produce diverse myelin protein zero neuropathies in transgenic mice. J Neurosci 2006; 26: 2358-2368.

12 Grandis M, Vigo T, Passalacqua $\mathrm{M}$ et al: Different cellular and molecular mechanisms for early and late-onset myelin protein zero mutations. Hum Mol Genet 2008; 17: 1877-1889.

13 Boylan KB, Ferriero DM, Greco CM, Sheldon RA, Dew M: Congenital hypomyelination neuropathy with arthrogryposis multiplex congenita. Ann Neurol 1992; 31: 337-340.

14 Nelis E, Timmerman V, De Jonghe P et al: Rapid screening of myelin genes in CMT1 patients by SSCP analysis: identification of new mutations and polymorphisms in the PO gene. Hum Genet 1994; 94: 653-657.

15 Senderek J, Hermanns B, Lehmann U et al: Charcot-MarieTooth neuropathy type 2 and P0 point mutations: two novel amino acid substitutions (Asp61Gly; Tyr119Cys) and a possible 'hotspot' on Thr124Met. Brain Pathol 2000; 10: 235-248.

16 Bellone E, Cassandrini D, Di Maria E et al: Novel MPZ mutation in a sporadic CMT patient. J Peripher Nerv Syst 2001; 6: 41.

17 Santoro L, Manganelli F, Di Maria E et al: A novel mutation of myelin protein zero associated with an axonal form of CharcotMarie-Tooth disease. I Neurol Neurosurg Psychiatry 2004; 75: $262-265$.

18 Bai I, Ianokova E, Pu Q et al: Effect of an R69C mutation in the myelin protein zero gene on myelination and ion channel subtypes. Arch Neurol 2006; 63: 1787-1794.

$19 \mathrm{Li} \mathrm{J}$, Bai Y, Ianakova E et al: Major myelin protein gene (P0) mutation causes a novel form of axonal degeneration. J Comp Neurol 2006; 10: 252-265.

20 Steck AJ, Erne B, Pareyson D, Sghirlanzoni A, Taroni F, SchaerenWiemers N: Normal expression of myelin protein zero with frame-shift mutation correlates with mild phenotypes.J Peripher Nerv Syst 2006; 11: 61-66. 